I'ja V. Gajduk

\title{
New York, 1960: Die Sowjetunion und die dekolonialisierte Welt auf der Fünfzehnten Sitzung der UN-Vollversammlung'
}

In der Geschichte des Kalten Krieges ist die Fünfzehnte Sitzung der UN-Vollversammlung als Wasserscheide zu sehen, mit Folgen nicht nur für die Vereinten Nationen selbst, sondern darüber hinaus für die gesamte Weltlage. Danach stand die Konfrontation der Großmächte mehr und mehr unter dem Einfluss von Faktoren, die ihren Ursprung in Regionen hatten, welche immer öfter als Dritte Welt bezeichnet wurden, also in jenem riesigen Gebiet, das außerhalb der eigentlichen Schlachtfelder des Kalten Krieges zwischen Ost und West lag, des Konflikts zwischen zwei „Welten“, die seit über einem Jahrzehnt um die Vorherrschaft rangen.

Was die Fünfzehnte Sitzung so herausragend erscheinen ließ, war das Zusammentreffen mehrerer Umstände, darunter die Aufnahme einer größeren Anzahl von Staaten in die Weltorganisation, von Staaten, die mit einer Ausnahme alle in Afrika lagen; dazu kam noch die Teilnahme ungewöhnlich vieler Staatsoberhäupter. Schließlich wurden auch Fragenkomplexe diskutiert, die sowohl für die Vereinten Nationen wie für die Weltgemeinschaft als Ganzes von Bedeutung waren. Zweifellos drückte die Teilnahme des sowjetischen Führers Nikita Chruščev den Debatten einen besonderen Stempel auf, ganz zu schweigen von seinem extravaganten Benehmen und seiner Hyperaktivität. Ihren Höhepunkt erreichte Chruščevs ungewöhnliche Form von Diplomatie am 12. Oktober 1960, dem Tag vor seiner Abreise. Erbost über Ausführungen des philippinischen Delegierten zur sowjetischen Unterdrückung Osteuropas, hämmerte Chruščev erst mit beiden Fäusten auf sein Pult; dann nahm er den Schuh zu Hilfe. Der peinlich berührte Gromyko folgte ihm mit weitaus weniger Kraft und Enthusiasmus. ${ }^{2}$

Aber wenn wir Chruščevs Hämmern mit Schuhen und Fäusten beiseitelassen, seine lauten Bemerkungen und seine unerwarteten Auftritte auf dem Podium, mit denen er seine Argumente möglichst kraftvoll vorbringen wollte, so ist der sowjetische Beitrag zum Verlauf und zu den Beschlüssen der Sitzung keineswegs zu unterschätzen. Dieser Beitrag ergab sich aus Wandlungen der sowjetischen Politik gegenüber der Außenwelt, vor allem gegenüber den unterentwickelten Ländern, aus Wandlungen, die dem Tod Stalins im Jahre 1953 folgten.

Solange Stalin lebte, schenkte Moskau den Vorgängen außerhalb Europas - von einigen Gebieten nahe den sowjetischen Grenzen abgesehen - wenig Aufmerksamkeit. Dies war ein unmittelbares Ergebnis sowohl der Stalin'schen Ansicht von den Prioritäten sowjetischer Außenpolitik nach dem Zweiten Weltkrieg als auch seiner Unterschätzung der nationalen Befreiungsbewegung in der Dritten Welt und ihres potentiellen Gewichts im Ringen zwischen der Sowjetunion und dem Westen. Die Theorie der „,wei Lager“ beherrschte das sowjetische Denken in der Frühphase des Kalten Krieges, und in dieser Theorie war kein Platz für nationalistische und neutralistische Sentiments, wie sie so viele asiatische und afrikanische Führer erfüllten, selbst jene, die sich als Kommunisten bezeichneten. Letztere

\footnotetext{
I Dieser Aufsatz wurde mit Unterstützung des Russischen Fonds für die Geistes- und Sozialwissenschaften erarbeitet (RGNF grant No.08-01-00)(58a).

"William Taubman, Khrushchev. The Man and His Era, New York 2003, S. 475f. und 657.
} 
waren zudem in ihrem Kampf um Unabhängigkeit mit ihrem nationalen Bürgertum verbunden, was im Kreml mit einigem Misstrauen betrachtet wurde, wenn das auch Anfang der fünfziger Jahre einen gewissen Wandel erfahren hatte, als Stalin auf dem XIX. Parteitag der KPdSU, der im Oktober 1952 stattfand, die These von der Unvermeidlichkeit eines Krieges zwischen der kommunistischen und der westlichen Welt aufgab; dieser sei weniger wahrscheinlich als Kriege zwischen kapitalistischen Ländern. Stalins Modifikation „öffnete, ideologisch gesehen, den Weg für die sowjetische Akzeptanz der Vielfalt der unterentwickelten Welt" ${ }^{\text {“3 }}$ und damit für eine andere Einstellung zu den Vereinten Nationen und anderen internationalen Organisationen, in denen Länder der Dritten Welt eine zunehmend wichtigere Rolle spielten.

Nikita Chruščev, dessen Machtübernahme mit einer neuen Welle der nationalen Befreiungsbewegungen zusammentraf, nahm die Anregung seines Vorgängers bereitwillig auf und entwickelte sie noch weiter im Rahmen seiner Gesamtkonzeption, die sowjetische Offenheit gegenüber der Außenwelt wollte und als „erste Détente“ bezeichnet wurde. So ist es nicht überraschend, dass seine ersten offiziellen Besuche in der nicht-kommunistischen Welt Ländern in Asien galten: Indien, Burma und Afghanistan. Die Reise nach Asien hinterließ bei dem sowjetischen Führer einen bleibenden Eindruck und zeigte ihm, dass die sowjetische Außenpolitik gähnende Lücken aufwies. Seitdem widmete Moskau den Vorgängen in Asien, Afrika und Lateinamerika mehr und mehr Aufmerksamkeit, auch nahm, auf verschiedenen Feldern, die Zusammenarbeit mit den Ländern jener Kontinente zu.

Zur Zusammenarbeit kam es sowohl auf der Ebene bilateraler Beziehungen wie durch internationale Organisationen, vor allem durch die Vereinten Nationen und ihr „Expanded Program of Technical Assistance (EPTA) “. Bereits im Juli 1953 gab der sowjetische Vertreter im Wirtschafts- und Sozialrat der UN bekannt, dass die UdSSR sich mit vier Millionen Rubel am UN-Programm für technische Unterstützung unterentwickelter Länder beteiligen werde. „Das brach mit einer langen Geschichte der Opposition gegen das Programm und signalisierte den Beginn einer größeren Anstrengung zur Umwerbung der Dritten Welt." ${ }^{\text {S }}$ Später wurden die Sowjets auch im Rahmen des „Special United Nations Fund for Economic Development (SUNFED) “ aktiv, der ins Leben gerufen worden war, um weniger entwickelten Ländern systematische und dauernde Hilfe für ihren technischen, wirtschaftlichen und sozialen Fortschritt zu sichern. Nach sowjetischen Schätzungen stellte die Sowjetunion den weniger entwickelten Ländern bis Anfang September 1959 Kredite in Höhe von 6,5 Milliarden Rubel (etwas mehr als eine Milliarde Dollar zum alten Rubelkurs) zur Verfügung. Zusätzlich gab Moskau Ländern wie Afghanistan, Kambodscha, Nepal und Indien 440 Millionen Rubel als unentgeltliche Hilfe."

Alles in allem hat die Sowjetunion - nach westlichen Schätzungen - in der Periode 1954 bis 1962 rund sechs Milliarden Dollar für Wirtschafts- und Militärhilfe in die Dritte Welt fließen lassen. ${ }^{\circ}$ Zwar bildeten die sowjetischen Beiträge zu EPTA und zum „Special Fund“ nur einen Bruchteil davon, da die sowjetische Hilfe vornehmlich über bilaterale Kontakte

\footnotetext{
${ }^{3}$ Alvin Z. Rubinstein, The Soviets in International Organizations: Changing Policy toward Developing Countries, 1953-1963, Princeton: Princeton University Press, 1964, S. 31.

${ }^{4}$ Bruce D. Porter, The LSSR in Third World Conflicts: Soviet Arms and Diplomacy in Local Wars, 1945-1980, Cambridge: Cambridge University Press, 1984, S. 16.

5 State Committec on Foreign Economic Ties to the CPSL CC, September 7, 1959, Rossijskij Gosudarstvennyj Archiv Novejšej Istorii (im Folgenden: RGANI), fond 5, opis' 30, delo 305, listy $123-124$.

"Rubinstein, The Soviets in International Organizations, S. 134.
} 
gewährt wurde, doch stellte Moskau die Rolle der UN bei der Entwicklungshilfe nicht mehr in Frage und zahlte in den UN-Fonds regelmäßig seine 4,7 Millionen Rubel (etwa 783000 Dollar) ein.

Offensichtlich verfolgte die Sowjetunion mit der Unterstützung der weniger entwickelten Länder vornehmlich den Zweck, das sowjetische Prestige zu erhöhen und Moskaus Position in der Dritten Welt zu stärken. In den Vereinten Nationen verschaffte das den Sowjets die Möglichkeit, jene Länder für die Unterstützung der Vorschläge und Resolutionen des Ostblocks zu gewinnen. Dass in die UN neue unabhängige Mitglieder aufgenommen wurden, von denen die meisten Kolonien gewesen waren, die ihre Freiheit eben erst errungen hatten und daher von anti-kolonialistischen und auch anti-westlichen Emotionen beherrscht waren, nährte in Moskau die Erwartung, die Kräfteverhältnisse in der Organisation zu seinen Gunsten verschieben und der westlichen Vorherrschaft, die so oft zur Überstimmung der Sowjetunion und ihrer wenigen Verbündeten geführt hatte, ein Ende machen zu können. Im Bezug auf die Vereinten Nationen löste so Optimismus den Skeptizismus und die Desillusionierung der Stalin'schen Jahre ab.

Die Tatsache, dass die meisten Führer der Dritten Welt ihren Neutralismus betonten, ihre Abneigung gegen eine Verwicklung in die Ost-West-Konfrontation, hat Chruščev und seine Mitarbeiter nicht entmutigt. Wohl war ihre Einstellung zum Neutralismus auf dem politischen Felde grundsätzlich negativ ${ }^{7}$, doch hingen sie fest dem Glauben an die Macht der Pragmatismen an, die die Neutralen in vielen Fragen, die auf UN-Ebene zu behandeln waren, zu einer Zusammenarbeit mit der Sowjetunion veranlassen werde. Dieser neue Standpunkt fand seinen Ausdruck im taktischen Verhalten der in den Vereinten Nationen tätigen sowjetischen Diplomaten. In der Stalin-Ära waren die Sowjets berüchtigt ob ihrer Arroganz gegenüber den Repräsentanten kleiner Nationen, zumeist der in Asien beheimateten, und ob ihrer Missachtung der Meinungen jener Repräsentanten ${ }^{8}$. In der zweiten Hälfte der fünfziger Jahre hingegen verfolgten sowjetische Delegierte eine subtilere Taktik, zu der „systematisches Lobbying“ der kleinen Nationen gehörte; persönliche Kontakte, Pressekonferenzen und Plaudereien in den Korridoren traten an die Stelle von Vernachlässigung und sogar offener Verachtung ${ }^{9}$.

Dass Moskau die Vereinten Nationen mehr und mehr als ein geeignetes Forum zur Gewinnung der Sympathien ehemaliger Kolonialvölker ansah, zeigte sich daran, dass die sowjetischen Führer die Rolle zu betonen begannen, die von der Weltorganisation bei der Sicherung der Rechte zu spielen sei, welche den früheren Kolonien und Semi-Kolonien im System der internationalen Beziehungen zukämen. Als Chruščev erstmals an einer Sitzung der UN-Vollversammlung teilnahm, im September 1959, erklärte er, die Vereinten Nationen seien der Ort, wo „ihr unveräußerliches Recht darauf, Herren ihres Geschicks zu sein“, gesichert werden könne. Es sei „die Pflicht der Vereinten Nationen“, so fügte er hinzu,

${ }^{7}$ Chruščev machte sich über den Antikommunismus der meisten neutralistischen Staaten keine Illusionen. In einem seiner Gespräche mit dem Inder Krishna Menon sagte er offen: „Aber neutralistische Länder sind nicht neutral. Sie sind gegen den Kommunismus [...]. Nicht alle, aber die Mehrheit." Siehe Prezidium CK KPSS, 1954-1964, Bd. 1: Černovye protokol'nye zapisi zasedanij. Stenogrammy, hrsg. von Aleksandr A. Fursenko, Moskau 2003, S. 506.

8 Siehe Beispiele hierzu in: Carlos P. Romulo, mit Beth Day Romulo, Forty Years: A Third World Soldier at the UN, Westport 1986.

9 Alexander Dallin, The Soviet Union at the United Nations: An Inquiry into Soviet Motives and Objectives, New York 1962, S. 100. 
„den äußersten Beitrag zum wirtschaftlichen Fortschritt der neuen Staaten zu leisten, die sich aus den Trümmern des kolonialen Systems erheben “. ${ }^{10}$

Wollen wir ein besseres Verständnis der Politik gewinnen, die Moskau in jenen Jahren gegenüber den früheren Kolonien verfolgte, müssen wir sehen, dass in den späten fünfziger Jahren die Sowjetunion nicht nur mit dem Westen um Einfluss in der Dritten Welt konkurrierte, sondern auch mit seinem chinesischen Verbündeten, der anfänglich indirekt, dann aber mehr und mehr offen den Anspruch der Sowjetunion anfocht, führender Protektor der nationalen Befreiungsbewegung und Sprecher der unterdrückten Völker zu sein. Wenn auch zur Zeit der Fünfzehnten Sitzung der UN-Vollversammlung die Differenzen zwischen Moskau und Beijing noch nicht offen ausgetragen wurden, so ließen die Chinesen doch keine Gelegenheit vorübergehen, ihre eigenständige Auffassung vom Ringen der Kolonialvölker um Unabhängigkeit und von der Rolle Chinas in diesem Kampf hervorzuheben."

Nach chinesischer Ansicht, wie sie 1961 von der sowjetischen Botschaft in Beijing berichtet wurde, stand China den Völkern Asiens, Afrikas und Lateinamerikas näher als die Sowjetunion und war daher das einzige Land, das ihnen effektive Hilfe leisten konnte. Seit 1953 hatte China insgesamt 250 Millionen Dollar an zehn Länder, darunter Burma, Indonesien, Guinea, Algerien und Kuba fließen lassen. ${ }^{12}$ Moskau beunruhigte aber weniger die Unterstützung, die China Entwicklungsländern gewährte; Anlass zur Sorge war vielmehr die 1959/60 deutlicher werdende Anstrengung Beijings, die führende Position der UdSSR als zuverlässiger Bundesgenosse im Befreiungskampf der Dritten Welt zu unterminieren. Die Botschaft registrierte die starke Zunahme der Kontakte zwischen den chinesischen Führern und Repräsentanten gerade unabhängig gewordener Staaten, die zahlreicher werdenden Reisen solcher Repräsentanten in die chinesische Hauptstadt und das chinesische Bemühen, den Besuchern zu schmeicheln und sie davon zu überzeugen, dass Beijing „das einzige Bollwerk der nationalen Befreiungsbewegung“ sei. Die Botschaft nannte mehr als neunzig Delegationen aus Entwicklungsländern, die Beijing allein im Jahre 1960 besuchten und zumeist von chinesischen Spitzenfunktionären willkommen geheißen wurden. ${ }^{13}$ Und die Botschaft vermerkte zugleich bitter, dass diese Funktionäre nur selten mit den Vertretern der Sowjetunion und anderer sozialistischer Staaten zusammenträfen.

\footnotetext{
${ }^{10}$ United Nations, Official Records of the General Assembly, Fourteenth Session, $799^{\text {th }}$ Plenary Meeting (September 18, 1959), zitiert in: George A. Brinkley, The Soviet Union and the United Nations: The Changing Role of the Developing Countries, in: The Review of Politics, Vol. 32, No. 1 (January, 1970), S.91-123, hier S. 98.

$11 \mathrm{Zu}$ den Differenzen zwischen der Sowjetunion und ihrem chinesischen Verbündeten von der frühen chinesisch-sowjetischen Allian $z$ bis zum offenen Zerwürfnis gibt es zahlreiche Literatur. Von den jüngeren Arbeiten zu diesem Thema sollen diejenigen, welche auf den in den letzten Jahren erschlossenen chinesischen Archivdokumenten basieren, erwähnt werden: Chen Jian, Mao's China and the Cold War, Chapel Hill 2001; Zhang Shuguang, Economic Cold War: America's Embargo against China and the Sino-Soviet Alliance, 1949-1963, Washington, D.C./Stanford 2001; Zhang Shuguang/Chen Jian, The Emerging Disputes between Beijing and Moscow: Ten Newly Available Chinese Documents, 1956-58, CWlHP Bulletin No. 6-7 (Winter 1995/1996). Zu dem sich anbahnenden Konflikt zwischen den zwei kommunistischen Riesen siehe auch folgende frühere Arbeit: Fejtö François, Chine-URSS. La fin d'une hégémonie: Les origins du grande schisme communiste, 1950-1957, Paris 1964.

${ }_{12}$ Bericht der sowjetischen Botschaft in der Volksrepublik China über die Haltung der Chinesischen Kommunistischen Partei gegenüber der Nationalen Befreiungsbewegung vom 29.4. 1961, RGANI, f.5, op. 49 , d. $435,1.45$.
}

${ }_{13}$ Ebenda, 1. 56. 
Die Sowjetunion wurde durch all das naturgemäß angereizt, in der Dritten Welt Sympathiewerbung zu betreiben, und zwar nicht nur durch Propaganda, sondern ebenso durch die Steigerung der Wirtschaftshilfe. Für die Propaganda stellten jedoch die Vereinten Nationen ein sehr geeignetes Forum dar; hier konnten im Kampf um das Herz und den Geist unterdrückter Völker Punkte gesammelt werden.

Die Dokumente zeigen, dass Moskau mit den Vorbereitungen für seine 1960 einsetzende Generaloffensive in den UN schon etliche Zeit zuvor begonnen hatte. Bereits 1958 betonte das Zentralkomitee der KPdSU in seinen Anweisungen zur Erweiterung der kulturellen und staatlichen Beziehungen mit den asiatischen und afrikanischen Ländern die Notwendigkeit, die sowjetische Beteiligung an den Aktivitäten des UN-Treuhänderschaftsrats sei zu nutzen, um die breite Öffentlichkeit „über die Situation in den vom Rat betreuten Ländern zu informieren und um die Interessen der Einwohner dieser Länder gegen den Kolonialismus zu verteidigen “. ${ }^{14}$ In Ausführung solcher Instruktionen richtete das sowjetische Außenministerium zum Beispiel an die sowjetische Vertretung bei den Vereinten Nationen Direktiven zur Frage der Zukunft Kameruns, die auf der Dreizehnten Sitzung der Vollversammlung diskutiert wurde. Nach den Direktiven sollte die Vertretung die Diskussion zur „kräftigen Darlegung unserer Einstellung zur nationalen Befreiungsbewegung der Völker Afrikas" nutzen. Die sowjetischen Delegierten erhielten die Weisung, Methoden der Unterdrückung und der Ausplünderung natürlicher Ressourcen, deren sich die Kolonialmächte bedienten, anzuprangern, ebenso die wahren Ziele der amerikanischen Politik in Afrika. Bei der Erfüllung dieser Aufgabe sei enge Verbindung mit „befreundeten Ländern Afrikas und Asiens“ zu halten, dazu mit „einigen lateinamerikanischen Delegationen“ und denen der sozialistischen Länder sowie Finnlands, Schwedens, Österreichs und Jugoslawiens; letztere wurden ausgewählt, weil sie nicht von der Sünde des Kolonialismus befleckt waren. ${ }^{15}$

In den Entwürfen der Direktiven für die Vierzehnte Sitzung der UN-Vollversammlung, ausgearbeitet von der sowjetischen Mission bei den Vereinten Nationen, nahmen Fragen des Kolonialismus einen der herausragenden Plätze ein. So wurde gesagt, Debatten über Treuhand-Gebiete, die auf der Tagesordnung der Sitzung standen, sollten genutzt werden, um eine „konstruktive Kritik“ an der Kolonialpolitik und -praxis von Staaten wie USA, England, Frankreich, Belgien usw. in Gang zu setzen. Auch wurde angeregt, andere Delegationen zur Initiierung „einer breiten Diskussion in der Vollversammlung über die politische Situation in Afrika, und zwar mit dem Ziel, zu einer Verurteilung sowohl der Unterdrückung im Kongo und in Njassaland wie der französischen Bemühungen um das sogenannte Franko-Afrikanische Commonwealth zu gelangen. ${ }^{16}$ Die sowjetische Mission machte auch folgenden Vorschlag: „Wenn man berücksichtigt, dass der Festlegung von Endterminen für die Erlangung der Unabhängigkeit der Treuhand-Gebiete eine besondere Bedeu-

14 ZK-Beschluss über Maßnahmen zur Ausweitung kultureller und gesellschaflicher Beziehungen mit den Ländern Asiens und Afrikas, Nr. St-61/35gs, 24.3.1958, RGANI, f. 4, op. 16, d. 469, 1. 73. Veröffentlicht in: Rossija i Afrika. Dokumenty i materialy, XVIII v. - 1960 g., hrsg. von A. B. Davidson und S. V. Mazov, Moskau 1999, Bd.2, S. 158-160.

15 MID, Direktive für die sowjetische Delegation zur 13. UN-Vollversammlung betr. die Zukunft der Treuhandgebiete unter französischer Verwaltung und des Treuhandgebiets Kamerun unter britischer Verwaltung, 12.2. 1959, Archiv Vnešnej Politiki Rossijskoj Federacii (im Folgenden: AVP RF), fond 047, opis' 5, papka 103, delo 16 , listy 5-6.

16 G. Arkadiev, amtierender ständiger Vertreter der UdSSR bei der UNO, an den stellvertretenden Außenminister Vasilij Kuznecov, „Streng Geheim“, 4.Juli 1959, beigefügt der Entwurf der Direktiven, ebenda, 1. 53. 
tung zukommt, so ist es notwendig, die Delegationen richtig anzufassen und, falls die ersten Versuche gute Resultate zeitigen, durch die Delegationen in afro-asiatischen Ländern oder zusammen mit ihnen eine Resolution vorzuschlagen, die für alle Treuhand-Gebiete, für eines nach dem anderen, feste Daten der Unabhängigkeit festsetzt.“ Auch solle die Resolution eine Klausel enthalten, die besagt, dass dieses Problem Thema einer eigenen Diskussion auf der Fünfzehnten Sitzung der Vollversammlung wird. ${ }^{17}$ Offenbar entsprach das der Stimmung der sowjetischen Führung: Als Nikita Chruščev an Bord des sowjetischen Dampfers „Baltika“ ging, um zu der Fünfzehnten Sitzung der UN-Vollversammlung zu reisen, nahm er den Auftrag mit, eine Unabhängigkeitserklärung kolonialer Länder und Völker zu präsentieren, die unter anderem Punkte enthielt, wie sie die sowjetische UN-Mission formuliert hatte.

Chruščevs Entscheidung, die Delegation persönlich zu leiten, war, ob spontan oder nicht, jedenfalls etwas unerwartet. Seine Absicht stand, engen Mitarbeitern zufolge, Mitte Juli 1960 fest, wurde aber erst am 10. August bekanntgegeben. Den sowjetischen Führer trieb ein starkes Verlangen, „sich für das zu rächen, was in Paris geschehen war“, eine Anspielung auf den Kollaps des Vier-Mächte-Gipfeltreffens, und die Leiter der westlichen Staaten zu zwingen, sich anzusehen, wie er vor aller Welt die USA und ihren Präsidenten demaskierte. ${ }^{18}$ Auch lockte ihn die Gelegenheit, öffentlich über seine Lieblingsthemen zu sprechen, über Abrüstung und Entkolonialisierung.

Es wurde erwartet, dass die Fünfzehnte UN-Vollversammlung für die Aufnahme von 17 neuen Mitgliedern stimmen werde, die meisten eben erst unabhängig gewordene afrikanische Staaten, und ein so erheblicher Zuwachs um ehemalige Kolonien musste der Entkolonialisierung ohnehin einen herausragenden Platz auf der Tagesordnung verschaffen. Die zwischen Ost und West bestehende Konkurrenz um Einfluss in den Entwicklungsländern und mithin um Einfluss auf deren Vertreter in der Vollversammlung gab dem Thema noch größere Bedeutung. So nahmen sich die Führer der beiden im Kalten Krieg rivalisierenden Blöcke - die der Vereinigten Staaten und die der Sowjetunion - vor, aus der Gelegenheit, ihren Beitrag zur Unabhängigkeit und Prosperität der ehemaligen Kolonien ins rechte Licht zu rücken, jedoch die Glaubwürdigkeit des Rivalen in Frage zu stellen, den äußersten Nutzen zu ziehen.

Als zum Beispiel das US State Department eine Generallinie vorbereitete, der die amerikanischen Führer in ihren Gesprächen mit Vertretern aus Afrika folgen sollten, nannte es unter anderem den Neutralismus afrikanischer Staaten, den die USA respektierten, wies aber zugleich warnend auf „die Notwendigkeit [hin], die Kontakte [dieser Länder] zum Sowjetblock und zum kommunistischen China zu kontrollieren“; ferner sprach es von der Rolle der afrikanischen Staaten in den Vereinten Nationen, die, so hoffte man in Washington, „verantwortungsbewusst“ sein werde, und von den Beziehungen zwischen Westeuropa und Afrika, die zu intensivieren seien; schließlich wurde die Hoffnung der USA erwähnt, „dass die afrikanischen Staaten sich hinsichtlich der militärischen Ausrüstung und Ausbildung an Westeuropa halten und Maßnahmen zur Regelung von Streitfragen wie zur Beschränkung der Rüstung und des Waffenimports erwägen werden “. ${ }^{19}$ Aus dem entsprechenden Dokument geht klar hervor, dass die Lenker der amerikanischen Politik wenig

17 Ebenda, 1. 35.

18 Taubman, Khrushchev, S. 472.

19 U.S. State Department, United States Policy on Africa (Basic Paper), Confidential, September 17, 1960, U.S. National Archives, RG-59, Conference Files, Box 236, CF 1770. 
Geschmack an der Neigung der neu etablierten Staaten zum Neutralismus fanden, aber sie mussten deren Recht zur Anknüpfung aller Arten von Beziehungen mit kommunistischen Ländern anerkennen und die wirtschaftliche und technische Hilfeleistung des Ostblocks oder Chinas als Faktum akzeptieren. Jedoch hielten sie es für angezeigt, die Führer jener Länder darauf aufmerksam zu machen, dass „strikte Kontrollen, das Prinzip der Gegenseitigkeit und die Vermeidung von Blockbeteiligung in sensitiven Bereichen essentiell“ seien. ${ }^{20}$ Natürlich unterstellte Washington bei seiner Warnung stillschweigend, dass der Westen und die ehemaligen Kolonien unter den „sensitiven Bereichen“ das Gleiche verstanden.

Es versteht sich, dass die Amerikaner nicht genau vorhersehen konnten, welche Taktik die sowjetische Delegation auf der Sitzung verfolgen werde, zumal unter der Leitung eines so unberechenbaren und impulsiven Mannes wie Chruščev. Der amerikanische Botschafter in Moskau, Llewellyn Thompson, sagte in einem Telegramm, das er am Vorabend der Sitzung nach Washington schickte, voraus, dass die Sowjetunion vor allem Abrüstung, die Vertretung Chinas und die amerikanische Aggressivität - wie sie in der Verletzung des sowjetischen Luftraums durch die U-2- und RB-47-Flüge gesehen werden könne - behandeln würde. Es schloss, Chruščev werde sich, allgemein gesagt, ,an eine Doppelstrategie halten und versuchen, die USA als kriegstreiberisch, provokant und imperialistisch hinzustellen, die UdSSR hingegen als friedliebend zu präsentieren“. ${ }^{21}$ Was dann tatsächlich geschah, scheint aber alle Erwartungen der amerikanischen Analysen übertroffen zu haben.

Chruščevs Äußerungen nach seiner Rückkehr aus New York machen klar, dass er von Anfang an beabsichtigte, die Fünfzehnte Sitzung der UN-Vollversammlung als Arena für eine groß angelegte Propaganda-Offensive Moskaus zu nutzen. Wie er am 20. Oktober 1960 in einer Rede vor Moskauern sagte: „Die sowjetische Regierung hielt es für erforderlich, dass die bedrängendsten, lebenswichtigen Probleme unserer Zeit auf der Sitzung diskutiert würden. "'22

Um das Propagandapotential ausschöpfen zu können, bestand er auf der Teilnahme der Führer der kommunistischen Länder an der Sitzung; er nahm zu Recht an, dass der Westen dann nicht umhin könne, als entsprechend nachzuziehen und ihm solchermaßen das größtmögliche Auditorium zu verschaffen. Vor diesem Publikum hielt Chruščev nicht weniger als elf Ansprachen mit Vorschlägen zur Abrüstung, zur Struktur der Vereinten Nationen und zum Kolonialismus. Einige westliche Beobachter schlossen daraus, dass die UN erstmals „selbst Fokus sowjetischer Politik waren, nicht nur Nebenaspekt anderer politischer Entscheidungen“".23 Und zum ersten Male verband Chruščev die beiden erstgenannten Themen mit der Frage der Entkolonialisierung.

Er machte diese Verbindung schon fast zu Beginn klar, als er in seiner großen Rede auf der Sitzung sagte: „Die Länder, die die Bürde des Kolonialismus abgeschüttelt haben, sind eine gewaltige und aktive Friedenskraft. Von nun an werden die jungen Staaten Afrikas und des Mittelmeerraums auch ihren hervorragenden Beitrag zur Lösung der wichtigen

\footnotetext{
20 Ebenda.

21 U.S. Department of State, Foreign Relations of the United States, 1958-1960, Vol. II: United Nations and General International Matters, Washington: United States Government Printing Office, 1991, S. 317.

22 Khrushchev in New York: A Documentary Record of Nikita S. Khrushchev's Trip to New York, September $19^{\text {th }}$ to October $13^{\text {th }}$, 1960, Including All His Speeches and Proposals and News Conferences, New York 1960, S. 242.

23 Dallin, The Soviet Union at the United Nations, S. 152.
} 
und komplizierten Probleme leisten, mit denen die Vereinten Nationen konfrontiert sind. " ${ }^{24}$ Und in den folgenden Passagen seiner über zweistündigen Rede erwähnte er mehr als einmal die wichtige Rolle, die den unabhängig gewordenen Ländern in der Weltarena zufallen werde. Er hob hervor, dass sie dazu beitragen würden, das Risiko militärischer Konflikte in der Welt zu verringern, er sprach von der „enormen Bedeutung, welche die Eliminierung des Kolonialregimes für die gesamte Weltwirtschaft" habe, er forderte die Umlenkung der Militärausgaben zur Steigerung der Hilfe für Entwicklungsländer, womit er den Nutzen der Abrüstung für die Dritte Welt unterstrich, und verlangte schließlich das war der sensationellste Part seiner Rede - eine Reform der UN-Struktur, die angezeigt sei, weil sich, neben den Blöcken der westlichen Mächte und der sozialistischen Staaten, die Gruppe der neutralistischen Staaten formiert habe, deren Interesse berücksichtigt werden müsste.

Chruščev zufolge war die Zeit für eine solche Reform umso mehr reif, als UN-Generalsekretär Dag Hammarskjöld „die Haltung einer lediglich formalen Verurteilung der Kolonialisten eingenommen " habe. Chruščev suchte den Vorwurf gegen den Generalsekretär mit dessen Verhalten in der Kongofrage, die ebenfalls im Mittelpunkt der Aufmerksamkeit der Vollversammlung stand, zu erhärten. Die seit dem 30. Juni 1960 von Belgien unabhängige Demokratische Republik Kongo hatte sich innerhalb von 14 Tagen mit weit um sich greifenden Soldatenunruhen, der als Schutzmaßnahme deklarierten Wiederkehr belgischer Truppen und der Sezession des kupferreichen Katangas konfrontiert gesehen. Das Zentrum war zudem bald durch den Machtkampf zwischen dem pro-westlichen Staatspräsidenten Joseph Kasavubu und dem pro-sowjetischen Ministerpräsidenten Patrice Lumumba paralysiert. Die bereits Mitte Juli 1960 herbeigerufenen UN-Truppen zeigten sich zunächst wenig geneigt, gegen die Sezessionisten vorzugehen, und die UN-Kommandeure vor Ort bevorzugten im Machtkampf der Präsidenten faktisch Kasavubu. ${ }^{25}$ Am 16. September schließlich putschte sich Joseph Desiré Mobuto an die Macht. Diese neue Konstellation war im Westen gern gesehen, zumal Mobutu am 17. September die sowjetische und die tschechische Botschaft schließen ließ. Vor solchem Hintergrund warf Chruščev Dag Hammarskjöld wütend vor, „die Linie der Kolonialisten“ zu vertreten. ${ }^{26}$

Zugleich bemühte sich Chruščev in seiner Rede, die Sowjetunion als die einzige aufrichtige und konsequente Helferin der unterdrückten Völker hinzustellen; sie sei frei von allen selbstsüchtigen Motiven und Hintergedanken. Um seine Behauptung zu beweisen, legte er den Entwurf einer Unabhängigkeitserklärung der Kolonialländer vor, der die Forderung enthielt, diesen Ländern sofort die vollständige Unabhängigkeit zu gewähren. ${ }^{27} \mathrm{Im}$ Laufe der Sitzung bestand die sowjetische Delegation darauf, dass die Deklaration auf Plenarsitzungen der Generalversammlung behandelt werde, nicht von ihrem Ersten (Politischen) Ausschuss.

Chruščevs Rede wirkte auf viele Delegierte wie eine Bombe. Die wachsende sowjetische Enttäuschung über die Behandlung der Kongo-Krise durch die Vereinten Nationen und ihren Generalsekretär war ja bereits bekannt. Zwar hatte Moskau für die Resolution gestimmt, die Hammarskjöld ermächtigte, eine friedenserhaltende Operation im Kongo ein-

24 Khrushchev in New York, S. 15.

25 Aleksandr Fursenko/Timothy Naftali, Khrushchev's Cold War. The Inside Story of an American Adversary, New York 2006, S. 307-318.

2ti Khrushchev in New York, S. 52.

27 Ebenda, S. 29. 
zuleiten, womit die UN einer Bitte der Regierung Lumumba entsprachen, doch begannen die Sowjets alsbald die Leitung der Vereinten Nationen zu kritisieren: Sie beweise zu viel Verständnis für die wirtschaftlichen Ziele des Westens in Afrika und für seine dortigen politischen Interessen im Rahmen des Kalten Krieges, zeige sich aber nicht gerade entschlossen, wenn es um Maßnahmen gehe, die erforderlich seien, um die Desintegration des Landes zu verhindern und belgische wie sonstige westliche Söldner daraus zu entfernen. Indes hatte niemand erwartet, dass solche Kritik Chruščev veranlassen werde, die Effizienz der UN-Struktur in Frage zu stellen und eine radikale Reform zu verlangen.

Am meisten Aufmerksamkeit fand natürlich Chruščevs Anregung, das Amt des UN-Generalsekretärs durch eine „Trojka“ zu ersetzen, bestehend aus Vertretern der drei Ländergruppen. Washington fasste Chruščevs Rede als Kriegserklärung an die Vereinten Nationen auf ${ }^{28}$; besondere Sorge galt den Reaktionen der Führer der Dritten Welt. Bald wurde aber klar, dass, entgegen Chruščevs Erwartungen, nur wenige von ihnen einer so radikalen Umgestaltung der Weltorganisation Beifall zollten. Den meisten kam der Vorschlag des sowjetischen Führers völlig überraschend, und sie befürchteten, dass diese Reform zur Zerstörung einer Institution führen könnte, an der sie nun ein Interesse hatten, weil dort die kleineren Nationen ihre Meinung zum Ausdruck und ihre Aspirationen zur Geltung bringen konnten. Westliche Delegationen spürten das. Als er mit Präsident Dwight D. Eisenhower zusammentraf, bemerkte der britische Premier Harold Macmillan: „Es herrscht der Eindruck, dass Chruščev sein Blatt wieder überreizt hat. "29

Alsbald machten die Führer der Dritten Welt den Amerikanern auch klar, dass sie von den Veränderungen, die Chruščev zur Diskussion gestellt hatte, in der Tat nicht allzu begeistert waren. Der indische Premier Jawaharlal Nehru sagte in einer Unterhaltung mit Eisenhower ganz offen: „Chruščevs Vorschlag, den Generalsekretär durch ein Triumvirat zu ersetzen, ist ,völlig undurchführbar “."30 Der ägyptische Präsident Gamal Abdel Nasser kritisierte zwar die Vereinten Nationen wegen ihrer Rolle im Nahost-Konflikt, erklärte aber in einem Gespräch mit Eisenhower ebenfalls, dass „es nicht nötig ist, zu debattieren, ob es eine UN geben soll oder nicht - die Vereinten Nationen werden offensichtlich gebraucht" . ${ }^{31}$ Seinerseits gab sich Eisenhower größte Mühe, die Bedeutung der UN in ihrer gegebenen Form hervorzuheben und Hammarskjölds Verhalten in der Kongofrage zu rechtfertigen.

Chruščev wiederum suchte die Führer der blockfreien Länder von der Richtigkeit seiner Ansichten zu überzeugen. Während seines Aufenthalts in New York traf er Nehru, Nasser, Kwame Nkrumah (Ghana) und Sékou Touré (Guinea), denen er die Argumente vortrug, die nach seiner Meinung für die Trojka sprachen. In seiner Konversation mit Nasser versicherte der sowjetische Partei- und Regierungschef, dass er nichts gegen Hammarskjöld persönlich habe; er sei, so Chruščevs Worte, „bei weitem nicht der schlechteste Kandidat für das Amt des Generalsekretärs“. Das System aber entspreche nicht der Lage in der Welt. „Amerika hat die Möglichkeit, von den Vereinten Nationen alles billigen zu lassen, was es

\footnotetext{
${ }^{28}$ Record of the Secretary of State's Staff Meeting, September 26, 1960, Foreign Relations of the United States (FRUS), 1958-1960, Vol. II, S.356.

29 Memorandum of a Conference with the President, September 27, 1960, ebenda, S.361.

30 Memorandum of Conversation, September 26, 1960, U.S. National Archives, RG-59, Conference Files, Box 235, CF 1767. Für eine veröffentlichte Version siehe: FRUS, 1958-1960, Vol. II, S. 357-359.

31 Memorandum of Conversation, Eisenhower-Nasser, September 26, 1960, U.S. National Archives, RG-59, Conference Files, Box 235, CF 1767.
} 
will“, so erklärte er. „Lasst uns diese unfaire Situation korrigieren.“32 Nassers vorsichtige Bemerkung, die Handlungen des Generalsekretärs würden von den ihm verliehenen Kompetenzen bestimmt und hingen davon ab, wer seine Entscheidungen ausführe, stieß offensichtlich auf taube Ohren; wieder und wieder warb Chruščev für die Trojka.

Die Pläne, Hammarskjöld abzuhalftern, die Kongo-Politik der UN zu ändern und dabei die Sympathien der unabhängig gewordenen Länder zu gewinnen, sind freilich am Ende gescheitert. Nicht einer der wichtigeren Bundesgenossen Moskaus in der Dritten Welt unterstützte eine Reform der UN nach den sowjetischen Vorschlägen. Selbst Nkrumah und Touré, scharfe öffentliche Kritiker des UN-Generalsekretärs, erwiesen sich nicht gerade als enthusiastische Befürworter einer radikalen Änderung der UN-Struktur. Sie traten lediglich für einen Kompromiss ein, demzufolge sichergestellt werden sollte, dass die Stellvertreter Hammarskjölds die drei Gruppen der Welt repräsentierten. ${ }^{33}$ Ein Jahr später gab Chruščev selbst, in einer Unterhaltung mit dem ägyptischen Botschafter, sein Scheitern zu, das er auf die Furcht der Entwicklungsländer vor dem Druck des Westens zurückführte. „Leider verstehen nicht alle Araber und nicht alle afrikanischen Neger unseren Standpunkt in dieser Frage“, vertraute er Botschafter Mohammed Galeb an. „Außerdem fürchten sich einige vor dem Imperialismus, der sie mit allen Mitteln einschüchtert. Ein Beispiel solcher Einschüchterung ist wohl die Weigerung der USA, Ghana Kredite für den Bau eines Dammes am Volta zu gewähren. “34 Nach Chruščevs Ansicht, die er dem ägyptischen Botschafter darlegte, sei es an der Zeit, in den Vereinten Nationen geheime Abstimmungen einzuführen; das gäbe afrikanischen und asiatischen Ländern mehr Freiheit, ihre Auffassungen zur Geltung zu bringen.

Etwas erfolgreicher waren die Sowjets mit ihrer Deklaration zum Kolonialismus. Wie beim Trojka-Vorschlag rechnete Moskau auch bei der Deklaration mit der vorbehaltlosen Zustimmung der unabhängig gewordenen Länder Afrikas und Asiens. In einer Studie, die in der Abteilung für internationale Organisationen des sowjetischen Außenministeriums entstand, gründete sich diese Erwartung auf die Tatsache, dass sich in den Vereinten Nationen eine Gruppe von Repräsentanten asiatischer, afrikanischer und arabischer Länder zusammengefunden hatte, die in vielen anti-kolonialistischen Fragen oft mit dem Ostblock stimmten. So nahmen die Sowjets zuversichtlich an, dass es ihnen gelingen werde, jene Länder für die Resolution zu gewinnen, zumal es möglich schien, dass der Westen versuchen werde, die sowjetische Resolution durch einen unverbindlichen Text zu ersetzen, in dem lediglich der Wunsch nach einer Beendigung des Kolonialregimes in ferner Zukunft zum Ausdruck komme. ${ }^{35}$

Solcher Argwohn Moskaus war nicht unbegründet. Tatsächlich umriss das State Department in Washington, als es die amerikanische Haltung in der Debatte über den sowjetischen Entwurf vorbereitete, drei Möglichkeiten, wie die Wirkung des sowjetischen Textes

32 Aufzeichnung des Gesprächs von N. S. Chruščev mit G. A. Nasser in New York vom 24.9. 1960, AVP RF, f.087, op. 21, p. 33, d.13, 11. 9-16. Veröffentlicht in: Bližnevostočnyi konflikt. 1957-1967: I7. dokumentov archiva vnešnei politiki Rossijskoj Federacii, Moskau 2003, Bd. 2, S. 336.

${ }^{33}$ Fursenko/Naftali, Khrushchev's Cold War, S. 318.

34 Aufzeichnung des Gesprächs von N. S. Chruščev mit dem Botschafter der UAR, Mohammed Galeb, am 9.10. 1961, in: Bližnevostočnyj konflikt, Bd.2, S. 366.

${ }^{35}$ MID, Abteilung Internationale Organisationen, „ढ̈ber die Taktik der sowjetischen Delegation in der Diskussion der Deklaration über die Gewährung der U'nabhängigkeit für koloniale Länder und Völker auf der XV.UN-Vollversammlung im September und Oktober 1960“, 20.2.1961, AVP RF, f. 0601, op. 2, p. 4, d. 10, 11. 67-76. Veröffentlicht in: Rossija i Afrika, Bd. 2, S. 174-178. 
auf die Entwicklungsländer abzuschwächen und seine Annahme in der Vollversammlung zu verhindern sei: Einbringung einer Gegen-Resolution, Zusätze zum sowjetischen Entwurf oder Sicherung ausreichender Stimmenthaltungen beziehungsweise ablehnender Voten. Von diesen Möglichkeiten, so das State Department, „empfiehlt sich vor allem die Einbringung einer gemäßigten Gegen-Resolution, sofern sie so gehalten werden kann, dass sie zumindest eine Zwei-Drittel-Mehrheit findet" ${ }^{36}$ Die Gegen-Resolution, meinte das State Department, solle von „freundlichen Afro-Asiaten“ eingebracht werden, unterstützt von europäischen Staaten wie Schweden, Norwegen, Irland und Österreich. ${ }^{37}$

Was den USA am sowjetischen Entwurf missfiel, war die Forderung, allen abhängigen Völkern die Unabhängigkeit sofort zu gewähren und Stützpunkte im Ausland abzubauen, ferner auch der Umstand, dass die Deklaration von der Sowjetunion vorgeschlagen wurde: Letzteres mochte einem Widersacher der Vereinigten Staaten einen Prestigegewinn verschaffen. Als eines der Argumente gegen die Glaubwürdigkeit des sowjetischen Vorschlags stellte Washington die Behauptung auf, die UdSSR sei selber eine solche Macht, „während andere ehemalige Kolonialreiche weitgehend liquidiert wurden " ${ }^{38}$ Nun konnte diese Behauptung von Osteuropäern und selbst von einigen Bewohnern zentralasiatischer Republiken der Sowjetunion günstig aufgenommen werden, doch war sie kaum geeignet, Entrüstung in den Herzen von afrikanischen Teilnehmern an der UN-Sitzung zu wecken. Was für sie am Kolonialismus Bedeutung hatte, war, wie Carlos Romulo, ein altgedienter philippinischer UN-Delegierter, sagte, „eine Frage der Hautfarbe“. „Die Bemühungen der Vereinigten Staaten, die Anklagen der Dritten Welt wegen Kolonialismus mit der Beschuldigung zu begegnen, die sowjetische Beherrschung Osteuropas sei ebenfalls Kolonialismus, stieBen in der Versammlung auf taube Ohren“, so schrieb er in seinen Memoiren, „vor allem weil die Länder der Dritten Welt den Kolonialismus als eine Frage der Farbe ansehen: weiße Herrschaft über schwarze und braune Völker. Die Unterdrückung von Weißen durch Weiße interessiert sie nicht. “39

Andererseits unternahmen die Sowjets Schritte, um zu erreichen, dass der sowjetische Entwurf, wenn er von der Vollversammlung schon nicht wörtlich akzeptiert wurde, nur solche Ergänzungen erfuhr, dass Moskau die Deklaration auch nach ihrer Modifizierung als sein Werk ausgeben konnte. Zu diesem Zweck richtete Chruščev, auf Vorschlag von Außenminister Andrej Gromyko, an die Führer afrikanischer UN-Mitglieder ein Schreiben, in dem er sie vor Intrigen der Westmächte gegen die Deklaration warnte. Er beschuldigte die Kolonialmächte des Versuchs, eine „klare und konkrete“ Entscheidung, das Kolonialsystem ungesäumt zu liquidieren, „durch eine überzuckerte Resolution, bar aller politischen Schärfe und Entschiedenheit“, zu ersetzen. ${ }^{40}$ Er gab der Hoffnung Ausdruck, dass

\footnotetext{
${ }^{36}$ State Department to U.S. Mission at the United Nations, November 1, 1960, FRUS, 1958-1960, Vol. II, S. 432.

${ }^{37}$ Bei den amerikanischen und sowjetischen Politikern ist eine ähnliche Denkweise festzustellen; auch Letztere sahen die Unterstützung von europäischen, vom Kolonialismus unbelasteten Ländern als wünschenswert an. Siehe oben über die sowjetischen Vorbereitungen zu der Fünfzehnten Sitzung der UN-Vollversammlung.

${ }^{38}$ Department of State to the U.S. Mission at the U.N., October 6, 1960, FRUS, 1958-1960, Vol.II, S. 399 .

${ }^{39}$ Romulo, Forty Years, S. 173.

40 Gromyko an das ZK der KPdSU, „Geheim“, 10. November 1960, AVP RF, f.0601, op. 1, p. 1, d.5, 1. 1b; N. S. Chruščev an Kwame Nkrumah, 12. November 1960, ebenda, f.573, op.4, p. 4, d.12, ll. 6-10; veröffentlicht in: Rossija i Afrika, Bd.2, S. 169-173. Identische Briefe waren an die Staats- und Regierungshäupter von weiteren 18 afrikanischen Ländern geschickt worden.
} 
die afrikanischen Führer die notwendigen Schritte unternehmen würden, einen solchen Gang der Dinge zu verhindern.

Als Resultat von Manövern, Intrigen, Verhandlungen hinter den Kulissen und unverblümtem Druck erhielten die Delegierten einen Resolutionsentwurf, den eine Gruppe asiatischer und afrikanischer Länder eingereicht hatte. Der Entwurf schien für beide Weltmächte akzeptabel zu sein. Die Sowjets fanden, dass er „im Grunde Prinzipien der sowjetischen Deklaration widerspiegelt, auch wenn er keinen Bezug auf den sowjetischen Entwurf nimmt". ${ }^{41}$ Zwar hatten die asiatischen und afrikanischen Autoren darauf verzichtet, ein festes Datum für die Gewährung der Unabhängigkeit zu nennen, doch waren die Sowjets bereit, in diesem Punkt ein Auge zuzudrücken. Das State Department wiederum konstatierte, der Entwurf sei „eine erhebliche Verbesserung früherer Entwürfe“. Außenminister Christian Herter meinte in einem Bericht an den staff secretary des Präsidenten, die Resolution „enthält viele Formulierungen, mit denen wir nicht übereinstimmen. Sie enthält aber auch etliche gute Formulierungen. ${ }^{42}$ Herter fügte hinzu, dass die amerikanische Delegation in New York „einhellig und entschieden für die Billigung der Resolution“ sei. ${ }^{43}$ Mit anderen Worten: Auch wenn weder Washington noch Moskau mit dem Entwurf ganz zufrieden waren, so fanden sie ihn doch annehmbar.

Moskau wies seine Delegation, nach der Einbringung zweier Zusätze, an, für die Resolution zu stimmen. Die Lage des amerikanischen Präsidenten war komplizierter. Er hatte ernste Bedenken gegen den Wortlaut der Resolution und befürchtete einen generell negativen Einfluss auf die amerikanische Politik. Es dauerte eine ganze Weile, bis ihn sein AuBenminister davon überzeugt hatte, dass „wir mitmachen sollten, da die Resolution ohnehin angenommen wird“. Obwohl Eisenhower eigentlich für Stimmenthaltung war, gab er schließlich seine Zustimmung. ${ }^{44}$ Jedoch musste er seine Entscheidung schon bald ändern. Einen Tag später erhielt er nämlich ein Schreiben des britischen Premierministers, der erklärte, für ihn sei es „ein Schock“, dass die Vereinigten Staaten beabsichtigten, für die afro-asiatische Resolution жu stimmen. ${ }^{45}$ Danach erhielt der Wunsch, einen europäischen Verbündeten zu beruhigen, Priorität vor der Absicht, in den Augen der Dritten Welt als Champion des Anti-Kolonialismus zu erscheinen. Noch am selben Tag rief Eisenhower Herter an und gab ihm die Weisung, sich der Stimme zu enthalten.

Die Stimmenthaltung der USA war der einzige unbestrittene Sieg, den die Sowjetunion auf der Fünfzehnten Sitzung der UN-Vollversammlung verbuchen konnte. Sie verursachte eine Welle der Empörung nicht nur unter den neutralistischen Ländern, sondern auch unter einigen europäischen Bundesgenossen der USA. Die amerikanische Mission bei den Vereinten Nationen, die durchweg dafür eingetreten war, der Resolution die amerikanische Stimme zu geben, ließ Washington einen detaillierten Bericht über Bemerkungen zukommen, die ausländische Delegierte gegenüber amerikanischen Kollegen gemacht hatten. ${ }^{46}$ "Wie konntet Ihr nur so stimmen? Ihr habt doch keine Probleme mit Kolonien“, verwunderte sich Alex Quaison-Sackey aus Ghana. Ein nigerianischer Delegierter fragte: „Versucht Ihr, politischen Selbstmord zu begehen?“ Ein Journalist aus Tunis äußerte sich

41 Wie Anm. 35, S. 177.

42 FRUS, 1958-1960, Vol. II, S. 450 und 454.

${ }_{43}^{4}$ Ebenda, S. 455.

${ }^{44}$ Memorandum of a Telephone Conversation between the President and the Secretary of State, December 8, 1960, ebenda, S. 456.

45 Siehe: Editorial Note, FRUS, 1958-1960, Vol. II, S. 457.

${ }^{46}$ U.S. Mission at the U.N. to State Department, December 15, 1960, ebenda, S. 460. 
besonders sarkastisch: „Glückwunsch zu Eurer Abstimmung! Wie ich höre, schickt Chruščev einen Orden." In der Tat hätte Moskau Anlass zum Feiern gehabt, als sich die USA mit so alten Kolonialisten wie den Engländern, Franzosen, Belgiern und Portugiesen zusammentaten. In seiner Aufzeichnung über die Diskussionen auf der Sitzung zog das sowjetische Außenministerium das Fazit: „Der Block der Kolonialisten, angeführt von den USA, befand sich in kompletter Isolierung." ${ }^{*} 7$

Aber diese Errungenschaft der Sowjetunion bei der Fünfzehnten Sitzung der UN-Vollversammlung war doch ziemlich oberflächlich. Entgegen den Hoffnungen der sowjetischen Führung, waren die neuen Drittwelt-Mitglieder der Weltorganisation weit davon entfernt, getreue Alliierte Moskaus bei dessen Aktivitäten im Rahmen der UN zu sein. Zwar hatte ihre Aufnahme in den Kreis der Vereinten Nationen die Lage innerhalb der Organisation deutlich verändert. Sie stimmten in vielen Fällen zusammen mit den Sowjets und deren kommunistischen Alliierten und die Westmächte stießen auf wachsende Schwierigkeiten bei der Suche nach Mehrheiten. Chruščev hatte sich jedoch erheblich verschätzt, als er annahm, die Entwicklungsländer seien naturgegebene Verbündete der UdSSR in der Friedensfrage und würden sich bei den meisten Problemen der Abrüstung, der wirtschaftlichen Zusammenarbeit, der rassischen Diskriminierung usw. automatisch dem kommunistischen Block anschließen. Sie zeigten sich in ihren Ausrichtungen beweglicher und in ihren Sympathien wie Antipathien wechselhafter, als man das in Moskau erwartet hatte. Schon die Fünfzehnte Sitzung der UN-Vollversammlung machte das deutlich. Als die Resolution zum Kolonialismus zur Entscheidung gestellt wurde, suchten einige Repräsentanten von Entwicklungsländern die sowjetische Delegation zu überreden, ihren Entwurf zurückzuziehen, da, wie sie sagten, „allein schon die Tatsache, dass eine Deklaration zur Liquidierung des Kolonialismus von der Sowjetunion vorgeschlagen wird, die Zustimmung aller afro-asiatischen Länder verhindert" “. ${ }^{8}$

Mit anderen Worten, die Dritte Welt stimmte nicht als ein monolithischer Block; sie war nicht notwendigerweise anti-westlich und pro-sowjetisch. Letzteres kann gut am Wechsel der für den jeweiligen Abstimmungssieger in den Sitzungen der UN-Vollversammlung abgegebenen Stimmen gezeigt werden. Die sowjetische „Erfolgsquote“ nahm von 42 Prozent im Jahre 1959 auf nahezu 46 Prozent in der wiederaufgenommenen Fünfzehnten Sitzung zu, die sich hauptsächlich mit afrikanischen Problemen befasste. Aber in der folgenden Sitzungsperiode, 1961, fiel sie auf 35 Prozent. Zwar stieg sie 1963, auf der Achtzehnten Sitzung, der letzten der Chruščev-Periode, wieder auf 44 Prozent, doch lag sie damit tiefer als zehn Jahre zuvor. ${ }^{49}$ Offenbar hatte Chruščevs Offensive auf der Fünfzehnten Sitzung, die auf die Konsolidierung einer "geschlossenen Front“ mit der entkolonialisierten Welt zielte, nur bescheidene Resultate erbracht, womit seine Nachfolger zu leben hatten, bis der Kalte Krieg zu Ende ging.

Aus dem Englischen übertragen von Dr. h. c. Hermann Graml

\footnotetext{
47 Wie Anm. 35, S. 178.

48 Ebenda, S. 175.

49 Brinkley, The Soviet Union and the United Nations: The Changing Role of the Developing Countries, in: The Review of Politics, Vol. 32, No. 1 (January, 1970), S. 102f.
} 